\title{
Rate of Breast Cancer Diagnoses among Postmenopausal Women with Self-Reported Breast Symptoms
}

\author{
Erin J. Aiello, MPH, Diana S. M. Buist, PhD, MPH, Emily White, PhD, Deborah Seger, \\ and Stephen H. Taplin, MD, MPH*
}

Background: Breast cancer rates in women with multiple breast symptoms have not been well described.

Methods: We examined the association between self-reported symptoms (lump, nipple discharge, pain, other) and breast cancer risk for screening and diagnostic mammograms in 57,681 women. Subanalyses evaluated risk among women with no prior mammograms, new symptoms, and repeated symptoms. One thousand, three hundred and eighty-nine women were diagnosed with cancer within 12 months of their mammograms. We calculated the breast cancer rate for each symptom and odds ratios (OR) with 95\% confidence intervals (CI) for breast cancer among symptomatic women compared with asymptomatic women.

Results: Women reporting a lump had an increased odds of breast cancer compared with asymptomatic women (OR for diagnostic examination $=2.8,95 \% \mathrm{CI}=2.3$ to 3.4 ; OR for screening examination $=3.6,95 \% \mathrm{CI}=2.6$ to 5.0 ). No other symptoms were associated with breast cancer after controlling for a reported lump. A new lump at a diagnostic examination was significantly predictive of cancer among women with no prior mammograms $(\mathrm{OR}=12.2,95 \% \mathrm{CI}=2.8$ to 53.5$)$; reporting symptoms at 2 successive exams had little effect on breast cancer risk $(\mathrm{OR}$ for lump $=1.2,95 \% \mathrm{CI}=0.6$ to 2.5$)$.

Conclusions: Having a lump is the most predictive symptom of breast cancer whether it is reported at a screening or diagnostic examination or in conjunction with other symptoms. ( $\mathrm{J}$ Am Board Fam Pract 2004;17:408-15.)

Approximately $70 \%$ to $80 \%$ of diagnostic mammographic examinations involve women who present with a breast problem; ${ }^{1,2}$ however, only $8 \%$ to $12 \%$ of these women go on to develop breast cancer. ${ }^{3-7}$ Although the prevalence of breast cancer diagnoses among women with symptoms is low, failure to diagnose breast cancer is one of the most common causes of malpractice claims. Malpractice claims are often associated with a story that the clinician ends

Submitted, revised, 9 August 2004.

From the Group Health Cooperative, Center for Health Studies (EJA, DSMB, DS, SHT), Department of Epidemiology, School of Public Health and Community Medicine, University of Washington (DSMB, EW), and Fred Hutchinson Cancer Research Center, Cancer Prevention Program (EW), Seattle. Address correspondence to Erin J. Aiello, Group Health Cooperative Center for Health Studies, 1730 Minor Avenue, Suite 1600, Seattle, WA 98101 (e-mail: aiello.e@ghc.org).

* Current address: Division of Cancer Control and Population Sciences, Applied Research Program, National Cancer Institute, Bethesda, MD.

This work was supported by grant CA63731 from the National Cancer Institute. a work-up of a palpable lump with a negative mammogram. ${ }^{8,9}$ Knowing which breast symptoms carry the highest breast cancer risk is important to assisting in the diagnosis of breast cancer. Most prior studies have not compared the proportion of breast cancer diagnoses in women with symptoms to a control group without symptoms; therefore, the relative importance of one symptom compared with others has not been well described.

Most previous studies have examined women who presented with symptoms at a diagnostic mammogram but not at a screening mammogram. ${ }^{3,5-7,10}$ Although screening is typically conducted among asymptomatic women, one study has reported that approximately $10 \%$ of women report symptoms at a screening examination. ${ }^{11}$ It is not clear how much the presence of symptoms increases the risk of breast cancer at screening exams. This is particularly important because studies have shown that the specificity of screening and diagnostic exams may be lower for women with breast symptoms compared with women without symptoms. ${ }^{1}$ 
Prior mammography and symptom history may influence the association between symptom occurrence and breast cancer. In one cross-sectional study, Barton et $\mathrm{al}^{12}$ showed that breast problems were reported at a rate of 23 examinations per 1000 person-years in a primary care setting. However, we are unaware of any longitudinal studies that have examined how often women present with the same symptoms that they have reported at prior exams and whether repeated symptoms are associated with an increase in breast cancer risk. It is not clear how the strength of any association between repeated symptoms and breast cancer compares with the association between new symptoms and breast cancer.

We examined the rate of breast cancer diagnoses among postmenopausal women who presented with or without breast symptoms at diagnostic and screening examinations using prospective data collected from Group Health Cooperative (GHC), a nonprofit integrated health system in western Washington. We determined the rate of breast cancer per year and adjusted odds ratios among symptomatic women compared with asymptomatic women. We also examined breast cancer rates among symptomatic women with no previous exams, new symptoms at a subsequent examination, and repeated symptoms at a subsequent examination compared with asymptomatic women.

\section{Methods}

\section{Study Population}

This was an observational study among female members of the staff-model component of an integrated health plan (GHC) that provides care to more than 500,000 people. All women were enrolled in GHC's Breast Cancer Screening Program (BCSP) from 1996 to $2001 .^{13-15}$ The BCSP began in 1986 to provide mammogram screening reminders and to track breast cancer risk factor information for women aged 40 years and older. The BCSP now corresponds with more than 80,000 women per year (approximately $85 \%$ of GHC women $\geq 40$ ) and screens more than 35,000 women per year in 6 screening centers. Women aged 40 to 49 years are offered screening every 1 to 2 years if they are at increased risk for breast cancer (classified by age at menarche, age at menopause, family history of breast cancer, breast biopsy history, and atypical hyperplasia). Women aged $\geq 50$ years are offered screening at 1- or 2-year intervals depending on their breast cancer risk status. The screening centers also provide diagnostic mammograms and ultrasounds. Risk factor information and symptoms are updated when women complete a paper survey at a mammography visit.

For the present study, we included the first screening and diagnostic mammographic exams for each woman in the BCSP per calendar year. Some women were included more than once in the population because they had more than one examination between 1996 and 2001. Thus, a total of 57,681 women received 123,847 total exams with complete symptom and risk factor data. We limited our analyses to postmenopausal women because of the different screening recommendations at GHC associated with age and the expected difference in rates of breast cancer associated with breast symptoms between pre- and postmenopausal women. ${ }^{13,14}$ Women were classified as postmenopausal if they reported no menstrual periods or were age 55 years or older. We excluded all short-interval follow-up exams, women with a previous history of breast cancer, and postcancer mammogram data for women who were diagnosed during the study period. Data collection was approved after requirements of GHC's Institutional Review Board.

\section{Data Collection}

We collected all demographic, risk factor, and symptom data from the BCSP survey, which is administered at the first mammogram in a calendar year for each woman. We collected information on demographics (age, ethnicity, and education level) and breast cancer risk factors (breast biopsy history, parity, age at menarche, current use of oral contraceptives, use of hormone therapy, family history of breast cancer, and body mass index). The radiologist's indication for the examination was used to determine the type of examination (screening or diagnostic) for each woman. If a woman had a diagnostic and screening examination in the same year, both were included in the analyses. If demographic or risk factor data were missing on the questionnaire for an examination, data from the previous questionnaire from that same woman were used to complete the missing data for items that were unlikely to change from one examination to the next (eg, age at menarche, education level). Information on breast cancer diagnoses was collected from the western Washington Surveillance, 
Epidemiology, and End Results (SEER) registry. We followed women for up to 1 year after their mammograms for a diagnosis of any in situ or invasive breast cancers.

We collected all symptom data from self-report on the BCSP survey at the time of the examination. Women noted whether they had any of the following breast problems in the 3 months before their examination: nipple discharge, lump, pain (only included on surveys from 1999 to 2001), or "other," which they were then asked to describe. Multiple symptom selections were allowed. We examined descriptions of "other" symptoms and reclassified some into the symptom categories described above (including pain for years before 1999). Other symptoms that were reported included having a rash, redness, moles or spots, swelling, change in size, itching, hormone-related problems, burning, thickening, bruising, skin changes, and implant problems. These individual symptoms were not analyzed separately because of their small numbers. All symptom information collected on the BCSP survey was available to radiologists when reading mammograms.

\section{Statistical Analyses}

We classified women with symptoms 2 different ways for the analyses. First, we classified women as symptomatic (having any symptom at the time of the examination) versus asymptomatic. Second, we evaluated symptom categories (ie, a lump or nipple discharge) compared with asymptomatic women. Women who presented with more than one symptom were included in the analysis for each. Asymptomatic women had no symptoms of any type.

We calculated the rate of breast cancer for the year after the mammogram. We also calculated the adjusted odds ratios (OR) and 95\% confidence intervals (CI) for breast cancer among symptomatic women compared with asymptomatic women using generalized estimating equations (GEE) with ordinary logistic regression. This approach accounts for women who had multiple mammograms during the study. We evaluated all demographic and risk factor variables as confounders by including them individually in the crude logistic regression model. Only those variables that changed the risk estimate by $10 \%$ or more were included in the final multivariate models. These covariates included breast biopsy history (yes, no), hormone therapy use (current, former, never, unknown), family history of breast cancer in a first- or second-degree relative (yes, no), body mass index (based on self-reported height and weight, continuous), and age at the time of the examination (continuous). We adjusted for the presence of a lump in additional analyses to determine the risk of breast cancer associated with breast symptoms beyond a lump. All analyses were stratified by type of examination (screening versus diagnostic). All statistical analyses were conducted using Stata 7SE (StataCorp., College Station, TX).

\section{Subanalysis to Examine Symptom History}

Prior mammogram and symptom history were collected for 40,939 women who were continuously enrolled in the BCSP from 1996 to 2001 and who had a mammogram between 1999 and 2001. Each woman was linked back to her most recent prior examination within 3 years of her current examination. Information on symptom history at the prior examination was gathered from the previous BCSP questionnaire for each woman. We classified mammogram and symptom history as follows: no prior mammograms within 3 years, prior mammogram with no symptoms, and prior mammogram with symptoms. We refer to women with a prior mammogram with symptoms as women with "repeated symptoms" even though the symptoms at each examination were not necessarily the same symptom. We could not accurately determine whether a lump reported at one examination was the same lump reported at a previous examination because all symptom data were from self-report and collected without reminding women of any previous symptoms. Therefore, we classified women's prior exams as having reported any prior symptoms or none, and not by the specific symptom reported. We calculated breast cancer rates and adjusted odds ratios for breast cancer among symptomatic women compared with asymptomatic women stratified by previous mammogram and symptom history using GEE with ordinary logistic regression. Odds ratios were adjusted for breast biopsy history, hormone therapy use, family history of breast cancer in a first- or second-degree relative, body mass index, and age at the time of the examination.

\section{Results}

We analyzed 13,524 diagnostic exams and 110,323 screening exams (Table 1). There were no differences in age, body mass index, or family history of 
Table 1. Characteristics of 57,681 GHC Women Who Obtained Diagnostic and Screening Mammograms between 1996 and 2001

\begin{tabular}{|c|c|c|c|c|}
\hline \multirow{3}{*}{ Number of women } & \multicolumn{2}{|c|}{$\begin{array}{c}\text { Diagnostic exams } \\
(\mathrm{N}=13,524 \text { exams })\end{array}$} & \multicolumn{2}{|c|}{$\begin{array}{c}\text { Screening exams } \\
(\mathrm{N}=110,323 \text { exams })\end{array}$} \\
\hline & \multicolumn{2}{|c|}{11,334} & \multicolumn{2}{|c|}{46,347} \\
\hline & Mean & SD & Mean & SD \\
\hline $\begin{array}{l}\text { Age } \\
\text { BMI }\left(\mathrm{kg} / \mathrm{m}^{2}\right)\end{array}$ & $\begin{array}{l}61.3 \\
27.2\end{array}$ & $\begin{array}{r}11.0 \\
6.3\end{array}$ & $\begin{array}{l}62.9 \\
27.2\end{array}$ & $\begin{array}{r}10.9 \\
6.4\end{array}$ \\
\hline & $\mathrm{N}$ & $\%$ & $\mathrm{~N}$ & $\%$ \\
\hline Family history of breast cancer & 5,112 & 37.8 & 40,378 & 36.6 \\
\hline $\begin{array}{l}\text { Hormone therapy use } \\
\text { Never } \\
\text { Former user } \\
\text { Current user } \\
\text { Unknown }\end{array}$ & $\begin{array}{l}1,300 \\
2,231 \\
2,479 \\
7,514\end{array}$ & $\begin{array}{l}21.6 \\
37.1 \\
41.2\end{array}$ & $\begin{array}{r}32,906 \\
19,446 \\
50,209 \\
7,762\end{array}$ & $\begin{array}{l}32.1 \\
19.0 \\
49.0\end{array}$ \\
\hline Previous biopsy & 3,513 & 26.0 & 22,603 & 20.5 \\
\hline Reported any symptom & 4,275 & 31.6 & 5,177 & 4.7 \\
\hline Diagnosed with breast cancer* & 666 & 4.9 & 723 & 0.7 \\
\hline $\begin{array}{l}\text { Prior mammogram history }{ }^{\dagger} \\
\text { No mammogram within } 3 \text { years } \\
\text { Mammogram without symptoms } \\
\text { Mammogram with symptoms }\end{array}$ & $\begin{array}{r}520 \\
4,645 \\
769\end{array}$ & $\begin{array}{r}8.8 \\
78.3 \\
13.0\end{array}$ & $\begin{array}{r}7,531 \\
25,792 \\
1,682\end{array}$ & $\begin{array}{r}21.5 \\
73.7 \\
4.8\end{array}$ \\
\hline
\end{tabular}

* Women were followed-up for 12 months after each exam for a breast cancer diagnosis.

${ }^{\dagger}$ Includes only women with exams from 1999 to 2001. N = 5,934 with diagnostic exams and 35,005 with screening exams.

breast cancer between women who had screening and diagnostic exams. Women with diagnostic exams were more likely to have used hormone therapy compared with women with screening exams (78\% former and current users vs $68 \%$, respectively). Women who had diagnostic exams were more likely to report symptoms compared with women who had screening exams $(32 \%$ vs $5 \%$, respectively). Subsequent to the 223,847 exams from 1996 to 2001, 1389 women (1.1\%) were diagnosed with breast cancer; a greater proportion was diagnosed after a diagnostic examination than after a screening examination ( $5 \%$ vs. $0.7 \%$, respectively).

There was a significant difference in the rate of breast cancer among women who received a diagnostic mammogram and reported any symptoms (66 per 1000 women per year) compared with asymptomatic women (41 per 1000 women per year, $P<$.001) (Table 2). Women who reported having any symptoms at a screening examination had a greater rate of breast cancer than women without symptoms (13 vs 6 per 1000 women per year, respectively). The most common symptom reported at a diagnostic examination was a lump. A reported lump was associated with the greatest breast cancer rates at both diagnostic (83 per 1000 women per year) and screening exams (22 per 1000 women per year). A reported lump was the only symptom associated with a significant increase in breast cancer risk at diagnostic and screening exams after adjustment for confounders $(\mathrm{OR}=2.8,95 \%$ $\mathrm{CI}=2.3$ to 3.4 , and $\mathrm{OR}=3.6,95 \% \mathrm{CI}=2.6$ to 5.0, respectively). Nipple discharge was associated with a small increase in the odds of breast cancer at screening and diagnostic exams; however, the increased odds disappeared when we adjusted these models for the reporting of a lump (data not shown). The presence of pain or other symptoms at screening or diagnostic exams was not associated with increased odds of breast cancer, with or without adjusting for a lump.

Among women with no prior exams, a lump was the most predictive symptom of breast cancer (breast cancer rate $=136$ per 1000 women per year) compared with asymptomatic women (15 per 1000 women per year) at a diagnostic examination (Table 3). Among women with prior exams, having a new lump was also more predictive of breast cancer compared with asymptomatic women. Women with repeated symptoms did not have higher rates of breast cancer compared with asymptomatic women at a diagnostic examination. At a screening examination, breast cancer rates were the 
Table 2. Breast Cancer Rates* and Adjusted Odds Ratios ${ }^{\dagger}$ (OR) with 95\% Confidence Intervals (CI) for Breast Cancer Associated with Breast Symptoms

\begin{tabular}{|c|c|c|c|c|c|c|c|c|}
\hline \multirow[b]{3}{*}{ Symptom $^{\ddagger}$} & \multicolumn{4}{|c|}{ Diagnostic Exams } & \multicolumn{4}{|c|}{ Screening Exams } \\
\hline & \multirow[b]{2}{*}{$\mathrm{N}$} & \multicolumn{2}{|c|}{ Breast Cancer } & \multirow[b]{2}{*}{ OR (95\% CI) } & \multirow[b]{2}{*}{$\mathrm{N}$} & \multicolumn{2}{|c|}{ Breast Cancer } & \multirow[b]{2}{*}{ OR (95\% CI) } \\
\hline & & $\mathrm{N}$ & Rate & & & $\mathrm{N}$ & Rate & \\
\hline None & 9,249 & 383 & 41 & 1.0 & 105,146 & 656 & 6 & 1.0 \\
\hline Any & 4,275 & 283 & 66 & $2.0(1.6,2.3)$ & 5,177 & 67 & 13 & $2.1(1.6,2.7)$ \\
\hline Lump & 2,745 & 229 & 83 & $2.8(2.3,3.4)$ & 1,740 & 38 & 22 & $3.6(2.6,5.0)$ \\
\hline Nipple discharge & 470 & 25 & 53 & $1.5(1.0,2.3)$ & 873 & 10 & 11 & $1.8(1.0,3.4)$ \\
\hline Pain & 287 & 15 & 52 & $1.4(0.8,2.4)$ & 694 & 6 & 9 & $1.5(0.7,3.4)$ \\
\hline Other & 1,400 & 58 & 41 & $1.2(0.9,1.6)$ & 2,672 & 20 & 7 & $1.2(0.8,1.9)$ \\
\hline
\end{tabular}

* Rate of breast cancer diagnoses per 1000 women per year.

${ }^{\dagger}$ Adjusted for age, body mass index, family history of breast cancer, previous breast biopsy, and hormone therapy use.

‡ Symptom categories are not mutually exclusive.

greatest among women with prior exams who had new symptoms compared with asymptomatic women. Similar patterns were noted after adjusting for confounders (Table 4). However, after we adjusted all the results for the reporting of a lump, only the odds ratio for nipple discharge among women with a diagnostic examination and no prior exams remained statistically significant $(\mathrm{OR}=8.8$, $95 \% \mathrm{CI}=1.7$ to 46.6 ) (data not shown).

\section{Discussion}

We examined the association between breast symptoms and breast cancer among a large population of postmenopausal women enrolled in a program de- signed to promote regular breast cancer screening. The presence of breast symptoms, especially a lump, is widely known to be associated with an increased risk of breast cancer, ${ }^{16}$ but the magnitude of the association is unclear. This large cohort of women allowed us to provide a more detailed description of the association between breast symptoms and risk of breast cancer, including an analysis among women with symptoms at screening mammograms, the impact of symptoms in addition to a lump, and modification of the association by prior symptom and mammogram history. To our knowledge, this is the first population-based report of the level of breast cancer risk associated with individual

Table 3. Breast Cancer Rates* among 40,939 Symptomatic and Asymptomatic Women Stratified by Diagnostic and Screening Exams, Prior Mammogram History, and Symptom History ${ }^{\dagger}$

\begin{tabular}{|c|c|c|c|c|c|c|c|c|c|c|c|c|}
\hline \multirow[b]{3}{*}{ Symptom $^{\ddagger}$} & \multicolumn{6}{|c|}{$\begin{array}{l}\text { Diagnostic Exams } \\
(\mathrm{N}=5,934)\end{array}$} & \multicolumn{6}{|c|}{$\begin{array}{l}\text { Screening Exams } \\
(\mathrm{N}=35,005)\end{array}$} \\
\hline & \multicolumn{2}{|c|}{$\begin{array}{l}\text { No Prior Exam } \\
\quad(\mathrm{N}=520)\end{array}$} & \multicolumn{2}{|c|}{$\begin{array}{l}\text { Prior Exam with } \\
\text { No Symptoms } \\
(\mathrm{N}=4,645)\end{array}$} & \multicolumn{2}{|c|}{$\begin{array}{c}\text { Prior Exam with } \\
\text { Symptoms } \\
(\mathrm{N}=769)\end{array}$} & \multicolumn{2}{|c|}{$\begin{array}{c}\text { No Prior } \\
\text { Exam } \\
(\mathrm{N}=7,531)\end{array}$} & \multicolumn{2}{|c|}{$\begin{array}{l}\text { Prior Exam with } \\
\text { No Symptoms } \\
(\mathrm{N}=25,792)\end{array}$} & \multicolumn{2}{|c|}{$\begin{array}{l}\text { Prior Exam with } \\
\text { Symptoms }^{\dagger} \\
(\mathrm{N}=1,682)\end{array}$} \\
\hline & $\mathrm{N}$ & $\begin{array}{l}\text { Breast } \\
\text { Cancer } \\
\text { Rate }^{*}\end{array}$ & $\mathrm{~N}$ & $\begin{array}{l}\text { Breast } \\
\text { Cancer } \\
\text { Rate }^{*}\end{array}$ & $\mathrm{~N}$ & $\begin{array}{l}\text { Breast } \\
\text { Cancer } \\
\text { Rate* }^{*}\end{array}$ & $\mathrm{~N}$ & $\begin{array}{l}\text { Breast } \\
\text { Cancer } \\
\text { Rate }^{*}\end{array}$ & $\mathrm{~N}$ & $\begin{array}{l}\text { Breast } \\
\text { Cancer } \\
\text { Rate }^{*}\end{array}$ & $\mathrm{~N}$ & $\begin{array}{c}\text { Breast } \\
\text { Cancer } \\
\text { Rate }^{*}\end{array}$ \\
\hline None & 132 & 15 & 3,885 & 45 & 248 & 61 & 7,142 & 3 & 24,714 & 2 & 1,394 & 5 \\
\hline Any & 388 & 111 & 760 & 101 & 521 & 48 & 389 & 3 & 1,078 & 9 & 288 & 10 \\
\hline Lump & 243 & 136 & 504 & 133 & 294 & 61 & 104 & 10 & 236 & 34 & 104 & 19 \\
\hline Nipple discharge & 44 & 182 & 72 & 42 & 65 & 31 & 69 & 0 & 146 & 7 & 62 & 16 \\
\hline Pain & 48 & 125 & 88 & 57 & 129 & 0 & 120 & 0 & 404 & 0 & 79 & 0 \\
\hline Other & 138 & 87 & 235 & 55 & 179 & 34 & 194 & 0 & 578 & 2 & 115 & 0 \\
\hline
\end{tabular}

* Rate of breast cancer diagnoses per 1000 women per year.

+ Symptoms reported at prior examination could have been any symptoms.

‡ Symptom categories are not mutually exclusive. 
Table 4. Adjusted* Odds Ratios (OR) with 95\% Confidence Intervals (CI) for Breast Cancer among Symptomatic Women Compared with Asymptomatic Women Stratified by Diagnostic and Screening Exams, Prior Screening History, and Symptom History ${ }^{\dagger}$

\begin{tabular}{lccccccc}
\hline & \multicolumn{3}{c}{ Diagnostic Exams } & & \multicolumn{3}{c}{ Screening Exams } \\
\cline { 2 - 3 } Symptom $^{\ddagger}$ & No Prior Exam & $\begin{array}{c}\text { Prior Exam with } \\
\text { No Symptoms }\end{array}$ & $\begin{array}{c}\text { Prior Exam with } \\
\text { Symptoms }\end{array}$ & & No Prior Exam & $\begin{array}{c}\text { Prior Exam with } \\
\text { No Symptoms }\end{array}$ & $\begin{array}{c}\text { Prior Exam with } \\
\text { Symptoms }\end{array}$ \\
\hline Any & $2.7(2.0,37.1)$ & $2.9(1.9,4.3)$ & $0.9(0.4,1.6)$ & & $1.0(0.1,7.4)$ & $4.5(2.3,8.9)$ & $2.1(0.5,8.3)$ \\
Lump & $12.2(2.8,53.5)$ & $4.1(2.7,6.2)$ & $1.2(0.6,2.5)$ & & $3.5(0.5,27.0)$ & $17.2(8.0,37.1)$ & $3.9(0.8,20.0)$ \\
Nipple discharge & $13.3(2.6,66.9)$ & $1.0(0.3,3.4)$ & $0.5(0.1,2.5)$ & & $3.0(0.4,22.1)$ & $3.8(0.5,33.0)$ \\
Pain & $8.9(1.6,50.0)$ & $1.5(0.6,4.0)$ & & & & $0.8(0.1,6.0)$ \\
Other & $6.4(1.4,29.7)$ & $1.5(0.8,2.9)$ & $0.6(0.2,1.6)$ & & &
\end{tabular}

* Adjusted for age, body mass index, family history of breast cancer, previous breast biopsy, and hormone therapy use.

† Symptoms reported at previous exam could have been any symptom.

‡ Symptom categories are not mutually exclusive.

and combined breast symptoms compared with asymptomatic women.

We found that women reported current symptoms at $32 \%$ of diagnostic exams and almost $5 \%$ of screening exams. These are lower than the prevalence of symptoms that has been previously reported among diagnostic and screening exams ( $70 \%$ to $80 \%$ and $10 \%$, respectively). ${ }^{1,2,11}$ One explanation for the lower prevalence of symptoms in our population may be that we included only postmenopausal women. The majority of other studies included premenopausal women as well, in whom the prevalence of breast symptoms is often much higher. ${ }^{16}$ However, not all studies have found such a high symptom prevalence among women with diagnostic exams. A study of 2400 women enrolled in a health maintenance organization found that only $16 \%$ had symptoms at a diagnostic examination, which is much lower than the rate that we found. ${ }^{12}$ That study based symptom reporting on physical findings by the physician, rather than a woman's self-report, which may have resulted in the lower prevalence of symptoms in that population compared with our study.

Likewise, the overall rate of breast cancer diagnoses associated with having any symptom was lower in our cohort than that found in other studies. In our study, $6.6 \%$ of women with symptoms at a diagnostic examination were diagnosed with breast cancer compared with $16 \%$ of women $>50$ years of age, as reported by Seltzer. ${ }^{4}$ The Seltzer study included women with symptoms or a prior abnormal mammogram (with or without symptoms present) that were referred to a private practice for a diagnostic examination. The inclusion of women with an abnormal examination may be why the Seltzer study noted a higher rate of breast cancer compared with our study. Although some of the women in our study may have had a prior abnormal examination, we categorized women solely on the presence of symptoms within 3 months prior or at the times of their examination.

We found that the rate of breast cancer among women with symptoms at a screening examination was also lower in our population (1.3\%) compared with one study of screening exams $(9.0 \%$, noted by Williams et $\mathrm{al}^{17}$ ). This difference may be because Williams et $\mathrm{al}^{17}$ evaluated only women with "significant" symptoms as defined by the Breast Test Wales (BTW) guidelines. ${ }^{18}$ The BTW considers any one of the following symptoms "significant": new lump; persistent, new localized pain; recent unilateral nipple retraction; recent unilateral nipple discharge; skin dimpling; or red, raw nipple.

Our findings of an increased risk of breast cancer associated with having a lump or nipple discharge are consistent with findings from other studies. ${ }^{12,19}$ Lumachi et al noted that having a lump was associated with a significantly increased risk of breast cancer among 884 women with symptoms over the age of 55 , yet the presence of nipple discharge was not. ${ }^{3}$ We noted no association between breast cancer risk and breast pain. This result was similar to that of Duijm et al, ${ }^{20}$ who found that only $0.4 \%$ of women who presented with pain at a screening mammogram were diagnosed with breast cancer.

We are unaware of any other epidemiologic studies that have examined the association between repeated symptoms and breast cancer risk. Our results showed that women who presented with any 
symptoms at 2 consecutive mammograms (screening or diagnostic) were not at an increased risk for breast cancer at the second examination. It is possible that the symptoms were stable and were not increasing in size or severity. However, we do not know whether women with a lump presented with the same lump at the previous examination and whether or not the lump(s) had changed. Repeated lumps may be fibroadenomas evaluated as benign that women may continue to report as a lump so that radiologists know about it when reading their mammograms. More than $50 \%$ of the women with repeated symptoms had additional evaluations after their previous mammograms. In addition, women who were diagnosed with cancer at a previous examination were excluded from this study. Therefore, the results for repeated symptoms may reflect symptoms that were previously evaluated as benign if the same lump or symptom was reported.

Symptoms were the most predictive of breast cancer among women getting a diagnostic examination who had no prior mammograms. We are unaware of any other studies that have examined the rate of breast cancer diagnoses among symptomatic women stratified by their prior examination history. It is possible that we saw a higher rate of breast cancer among these women compared with women with prior exams because the rate included prevalent cancers rather than incident cancers. Although women with no prior exams comprised less than $9 \%$ of our study population who had a diagnostic examination, the high rate of breast cancer in this group may warrant extra consideration when evaluating mammograms of symptomatic women.

The evaluation of breast symptoms is a common occurrence in primary care that often leads to a mammogram and much less often leads to a cancer. Only $8 \%$ to $12 \%$ of symptomatic women develop cancer ${ }^{3-7}$, so clinicians must exercise some judgment about how to evaluate symptomatic women. It is clear from this study that a self-reported breast lump is associated with an increase in breast cancer risk at both diagnostic and screening exams; the largest increase in risk occurs among women with no prior mammograms. Women with concerns about lumps need careful evaluation, including aspiration, ultrasound, or biopsy, regardless of the mammogram result if the lump is palpable. ${ }^{8,9}$ What may be more important about these results is that they provide some assurance that other symptoms are much less likely to be associated with cancer. This does not mean that those symptoms should be ignored; rather, observation and re-evaluation may be acceptable approaches if the symptoms resolve with time and no lump develops.

Information regarding symptoms may be useful for radiologists to have as they review mammograms. It is not clear from the literature whether radiologists have patient symptom information when reviewing diagnostic or screening films. In our study, radiologists had the patient's self-reported symptom data available when reviewing diagnostic and screening exams because these data are routinely collected during the mammogram appointment. However, this may not be the case in every mammography clinic. To maximize detection, it may be important to systematically collect symptom information on all women, but the contribution of this information to detection needs closer evaluation.

The fact that all women were enrolled in the population-based BCSP was one of the major strengths of our study. This is the largest prospective study so far on self-reported symptoms and breast cancer risk. We were able to limit our study population to postmenopausal women, who have a greater risk of breast cancer associated with symptoms than premenopausal women. ${ }^{16}$ In addition, we were able to collect a wide variety of symptom data, including information on symptoms at screening exams, multiple symptoms, and symptom occurrence at prior exams. We were able to adjust the statistical models for multiple mammography examinations during the study time period. However, the results of our study may not be generalizable to other populations that are not receiving screening mammography on a consistent schedule. Our study population was predominantly white, and all women received their care as members of a health maintenance organization. It is possible that symptom characteristics and breast cancer rates may be different among more ethnically diverse populations.

In conclusion, our data showed that the presence of a lump was associated with a 2 - to 3 -fold greater risk of breast cancer with and without the presence of any other symptoms at both screening and diagnostic mammograms. The risk of breast cancer associated with symptoms, particularly a lump, was amplified among women with no prior exams, but not among women with symptoms at the prior 
examination. Women who reported pain or "other" symptoms did not seem to be at increased breast cancer risk. Our findings reinforce the importance of fully evaluating a reported lump, and should reassure physicians that careful observation of women with nipple discharge and pain may be justified.

\section{References}

1. Barlow WE, Lehman CD, Zheng Y, et al. Performance of diagnostic mammography for women with signs or symptoms of breast cancer. J Natl Cancer Inst 2002;94:1151-9.

2. Geller BM, Barlow WE, Ballard-Barbash R, et al. Use of the American College of Radiology BI-RADS to report on the mammographic evaluation of women with signs and symptoms of breast disease. Radiology 2002;222:536-42.

3. Lumachi F, Ermani M, Brandes AA, et al. Breast complaints and risk of breast cancer. Populationbased study of 2,879 self-selected women and longterm follow-up. Biomed Pharmacother 2002;56: $88-92$.

4. Seltzer MH. The significance of breast complaints as correlated with age and breast cancer. Am Surg 1992;58:413-7.

5. Dawson C, Lancashire MJ, Reece-Smith H, Faber RG. Breast disease and the general surgeon. I. Referral of patients with breast problems. Ann R Coll Surg Engl 1993;75:79-82.

6. Locker AP, Manhire AR, Stickland V, Caseldine J, Blamey RW. Mammography in symptomatic breast disease. Lancet 1989;1:887-9.

7. Lumachi F, Ermani M, Brandes AA, et al. Prevalence of breast cancer in women with breast complaints. Retrospective analysis in a population of symptomatic patients. Anticancer Res 2002;22:3777-80.

8. Kerlikowske K, Smith-Bindman R, Ljung BM, Grady D. Evaluation of abnormal mammography results and palpable breast abnormalities. Ann Intern Med 2003;139:274-84.
9. Donegan WL. Diagnosis of breast cancer. In: Donegan WL, Spratt JS, eds. Cancer of the breast, 5th ed. Philadelphia: WB Saunders; 2002. p. 311-46.

10. Seltzer MH, Glassman JR. Benign-appearing mammographic abnormalities in women aged 40-49. Breast J 2002;8:162-70.

11. Kavanagh AM, Giles GG, Mitchell H, Cawson JN. The sensitivity, specificity, and positive predictive value of screening mammography and symptomatic status. J Med Screen 2000;7:105-10.

12. Barton MB, Elmore JG, Fletcher SW. Breast symptoms among women enrolled in a health maintenance organization: frequency, evaluation, and outcome. Ann Intern Med 1999;130:651-7.

13. Taplin SH, Thompson RS, Schnitzer F, Anderman C, Immanuel V. Revisions in the risk-based Breast Cancer Screening Program at Group Health Cooperative. Cancer 1990;66:812-8.

14. Carter AP, Thompson RS, Bourdeau RV, Andenes J, Mustin H, Straley H. A clinically effective breast cancer screening program can be cost-effective, too. Prev Med 1987;16:19-34.

15. Taplin SH, Ichikawa L, Buist DS, Seger D, White E. Evaluating organized breast cancer screening implementation: the prevention of late-stage disease? Cancer Epidemiol Biomarkers Prev 2004;13:225-34.

16. Morrow $\mathrm{M}$. The evaluation of common breast problems. Am Fam Physician 2000;61:2371-8, 2385.

17. Williams RS, Brook D, Monypenny IJ, GowerThomas K. The relevance of reported symptoms in a breast screening programme. Clin Radiol 2002;57: 725-9.

18. Breast Test Wales. Quality Manual. 96. Cardiff: Breast Test Wales.

19. Gulay H, Bora S, Kilicturgay S, Hamaloglu E, Goksel HA. Management of nipple discharge. J Am Coll Surg 1994;178:471-4.

20. Duijm LE, Guit GL, Hendriks JH, Zaat JO, Mali WP. Value of breast imaging in women with painful breasts: observational follow up study. BMJ 1998; 317:1492-5. 